\title{
Una aplicación de la solución de sistemas de ecuaciones lineales no homogéneas con matriz en banda, obtención de la elástica de barras de eje recto
}

\author{
F. Monroy-Miranda \\ Profesor de la Facultad de Ingeniería, UNAM
}

(recibido: agosto de 1996; aceptado: enero de 2001)

\begin{abstract}
Resumen
En ingeniería estructural, la obtención de la elástica de una barra es uno de los problemas en donde hay que resolver un sistema de ecuaciones con arreglo de matriz en banda, cuando la ecuación que la rige se ha expresado utilizando el método de diferencias finitas. En este trabajo se presenta un resumen del método y se desarrolla un programa de computadora que incorpora la solución del sistema de ecuaciones mediante la aplicación del procedimiento presentado en el artículo: "Solución de sistemas de ecuaciones lineales no homogéneas con matriz en banda", publicado en 1996 en esta revista. Con este programa se resuelven algunos ejemplos y uno de ellos se compara con la solución exacta.
\end{abstract}

Descriptores: ecuaciones, lineales, homogéneas, matriz, banda, elástica, barra.

\section{Abstract}

Obtainment of the elastic line of a bar in structural engineering is one of the problems where it is necessary to solve a set of equations arranged in a band matrix, when the equation that governs it has been stated in terms of the finite difference method. This paper presents a summary of the method and the development of a computer program incorporating the solution to the set of equations through the application of the procedure disclosed in the article entitled "Solution of non-homogeneous linear equations with band matrix" published in 1996 in No. 2 issue of this journal; some problems have been solved with this program and in one of them a comparison is made of the results with the exact solution.

Keywords: homogeneous linear equations, band matrix, elastic line, bar.

\section{Introducción}

Las microcomputadoras de hoy en día han revolucionado muchas de las actividades de la ingeniería civil, sobre todo, si se aprovecha la interacción entre el usuario y la computadora, así como la capacidad de graficación de la pantalla, lo cual resulta adecuado para muchas de las actividades relacionadas con la práctica actual de esta materia. Uno de los problemas que surgió con la aparición de la computadora digital, fue el manejo y la interpretación por parte del usuario de los resultados numéricos proporcionados por la misma, más aún, cuando la cantidad de éstos resultaba considerable, como sucede con varios de los problemas de ingeniería estructural.

Un caso concreto de lo anterior, lo constituyen los desplazamientos de puntos de una estructura producidos por un sistema de fuerzas actuando sobre ella, que son una parte de los resultados proporcionados por casi todos los métodos y programas de computadora para análisis estructural. Sin lugar a dudas, el problema se resuelve cuando después de que los resultados han sido expresados numéricamente se hace de manera gráfica, con las ventajas que esto representa.

En el caso de la elástica, existe un inconveniente, por razones prácticas sólo son considerados en el análisis, la 
menor cantidad de datos necesarios para definir completamente a la estructura, trayendo como consecuencia que no se pueda dibujar con todo rigor la elástica de la misma con los desplazamientos de los nudos definidos (nudos extremos de la barra), o bien, se haga de manera aproximada, ya que para la mayoría de las barras no son conocidos los desplazamientos de puntos intermedios.

Si se introducen más nudos, se requerirá de más esfuerzo en la preparación de datos, así como tiempo y memoria de la computadora para la solución del problema, factor que en algunos casos, todavía es determinante en el uso de algunos programas de cómputo para la solución de ciertos problemas.

La solución que se presenta a continuación, consiste en aplicar la ecuación de la elástica a cada barra, tomando como condiciones de frontera los desplazamientos de sus extremos obtenidos del análisis de la estructura; resolviendo la ecuación de la elástica se obtienen los desplazamientos de puntos intermedios de la barra. Ahora, se resume la teoría requerida y se presenta un programa de computadora para la obtención de la elástica de barras planas de eje recto, considerando solamente efectos de flexión; asimismo, incluye su representación gráfica.

Planteamiento matricial de la ecuación de la elás tica de una barra en el plano, considerando sólo efectos de flexión

La aplicación de la mecánica de materiales permite obtener la ecuación que define la elástica de una barra sujeta a fuerzas contenidas en su plano (figura 1), ésta se logra mediante dos integraciones sucesivas partiendo de la siguiente ecuación diferencial.

$$
\frac{d^{2} y}{d x^{2}}=\frac{M}{E I}
$$

En la ecuación anterior:

$\mathrm{y}=$ flecha en cualquier punto de la barra.

$\mathrm{x}=$ distancia variable a lo largo del eje de la barra.

$\mathrm{M}=$ momento flexionante en cualquier punto a lo largo del eje de la barra.

$\mathrm{E}=$ módulo elástico del material que forma a la barra.

$\mathrm{I}=$ momento de inercia de área de la sección transversal de la barra con respecto al eje de flexión.

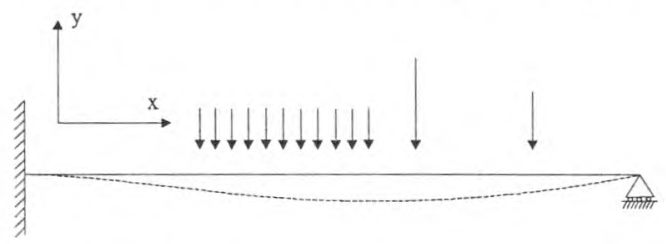

Figura 1. Elástica de una barra
En general, el cociente M/EI puede ser expresado mediante una o varias funciones continuas de la distancia variable $\mathbf{x}$ a lo largo del eje de la barra, cuando esa relación se puede obtener fácilmente, el método de la doble integración puede constituir un procedimiento conveniente para ser utilizado manualmente y de esta manera, determinar analíticamente la ecuación de la elástica. Sin embargo, si el número y tipo de fuerzas que actúan sobre la barra es considerable, o si el producto EI varia a lo largo de la barra, la obtención de la elástica puede volverse tediosa (aun utilizando funciones de singularidad o algún otro método), sobre todo si se pretende plantear para ser resuelta utilizando la computadora.

La reducción de la ecuación (1) a la forma de diferencias finitas con el conjunto resultante de ecuaciones lineales algebraicas, suministra un método conveniente para ser programado en una computadora digital. Utilizando la primer expresión de las diferencias centrales correspondientes a $\mathrm{d}^{2} \mathrm{y} / \mathrm{d} \mathrm{x}^{2}$, la ecuación (1) se puede escribir de la siguiente forma:

$$
\frac{y_{i+1}-2 y_{i}+y_{i-1}}{(\Delta x)^{2}}=\left[\frac{M}{E I}\right]
$$

O bien

$$
y_{i+1}-2 y_{i}+y_{i-1}=\left[\frac{M}{E I}\right]_{i}(\Delta x)^{2}
$$

Donde el subíndice de (M/EI) indica que es una función de $\mathbf{x}$, aplicando la ecuación (3) en los puntos 2 a N-1, siendo $\mathrm{N}$ el número de puntos considerados a lo largo del eje de la barra se obtiene el siguiente sistema de ecuaciones algebraicas:

$$
y_{1}-2 y_{2}+y_{3}+0 y_{4}+\ldots \ldots \ldots . . .0 y_{n}=\left[\frac{M}{E I}\right]_{2}(\Delta x)^{2}
$$

$$
\begin{aligned}
& 0 y_{1}+y_{2}-2 y_{3}+y_{4}+0 y^{5}+\ldots . .0 y_{n}=\left[\frac{M}{E I}\right]_{3}(\Delta x)^{2} \\
& 0 y_{1}+0 y_{2}+y_{3}-2 y_{4}+y_{5}+\ldots . .0 y_{n}=\left[\frac{M}{E I}\right]_{4}(\Delta x)^{2}
\end{aligned}
$$

$$
0 y_{1}+\ldots+0 y_{n-3}+y_{n-2}-2 y_{n-1}+y_{n}=\left[\frac{M}{E I}\right]_{n-1}(\Delta x)^{2}
$$

El sistema anterior de $\mathrm{N}-2$ ecuaciones algebraicas lineales contiene $\mathrm{N}$ términos que denotan las flechas $\mathrm{y}_{1}, \mathrm{y}_{2}$, $\ldots y_{n}$; no obstante, por ser condiciones de frontera se conocen los valores $y_{1}$ y $y_{n}$, en consecuencia, las ecuaciones 
(4) constituyen un conjunto de $\mathrm{N}-2$ ecuaciones independientes que contienen $\mathrm{N}-2$ incógnitas, las cuales se expresan de la siguiente manera:

$$
\begin{gathered}
y_{2}-\frac{1}{2} y_{3}+0 y_{4}+\ldots . .0 y_{n-1}=-\frac{1}{2}\left[\frac{M}{E I}\right]_{2}(\Delta x)^{2}+\frac{1}{2} y_{1} \\
-\frac{1}{2} y_{2}+y_{3}-\frac{1}{2} y_{4}+0 y_{5}+\ldots .0 y_{n-1}=-\frac{1}{2}\left[\frac{M}{E I}\right]_{3}(\Delta x)^{2}
\end{gathered}
$$$$
O y_{2}-\frac{1}{2} y_{3}+y_{4}-\frac{1}{2} y_{5}+\ldots .0 y_{n-1}=-\frac{1}{2}\left[\frac{M}{E I}\right]_{4}(\Delta x)^{2}
$$

$$
0 y_{2}+\ldots .0 y_{n-3}-\frac{1}{2} y_{n-2}+y_{n-1}=-\frac{1}{2}\left[\frac{M}{E I}\right]_{n-1}(\Delta x)^{2}+\frac{1}{2} y_{n}
$$

Finalmente, en forma matricial :

$$
[\mathrm{A}]\{\mathrm{Y}\}=\{\mathrm{X}\}
$$

Donde:

$$
X=\left\{\begin{array}{c}
-\frac{1}{2}\left[\frac{M}{E I}\right]_{2}(\Delta x)^{2}+\frac{1}{2} y_{1} \\
-\frac{1}{2}\left[\frac{M}{E I}\right]_{3}(\Delta x)^{2} \\
-\frac{1}{2}\left[\frac{M}{E I}\right]_{4}(\Delta x)^{2} \\
\cdot \\
-\frac{1}{2}\left[\frac{M}{E I}\right]_{n-1}(\Delta x)^{2}+\frac{1}{2} y_{n}
\end{array}\right]
$$

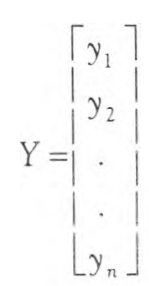

Los valores de (M/EI) en cada punto, al igual que los valores del desplazamiento $y_{1}$ y $y_{n}$ correspondientes a las condiciones de frontera deben ser conocidos.

Finalmente, la solución del sistema de ecuaciones simultáneas (5) proporciona valores únicos para las flechas deseadas.

\section{Desarrollo del programa}

Con lo expuesto anteriormente, se desarrolló el programa ARTIC3 en QUICK-BASIC versión 4.0 (lenguaje BASIC bastante mejorada), el programa incluye la representa. ción gráfica de los desplazamientos perpendiculares al eje de la barra (dibujo de la elástica). En cuanto a la solución del sistema de ecuaciones, uno de los métodos aplicables es el de eliminación de Gauss, adaptado al caso de sistemas con matrices en banda, este método se expuso en el número 2 de 1996 de esta publicación, del cual se modificó el programa SSELMB para ser utilizado en este trabajo.

\section{Ejemplos}

Para la solución de cada uno de los siguientes ejemplos se preparó el archivo de datos correspondiente para luego ejecutarse el programa, datos, resultados y listado del programa. 


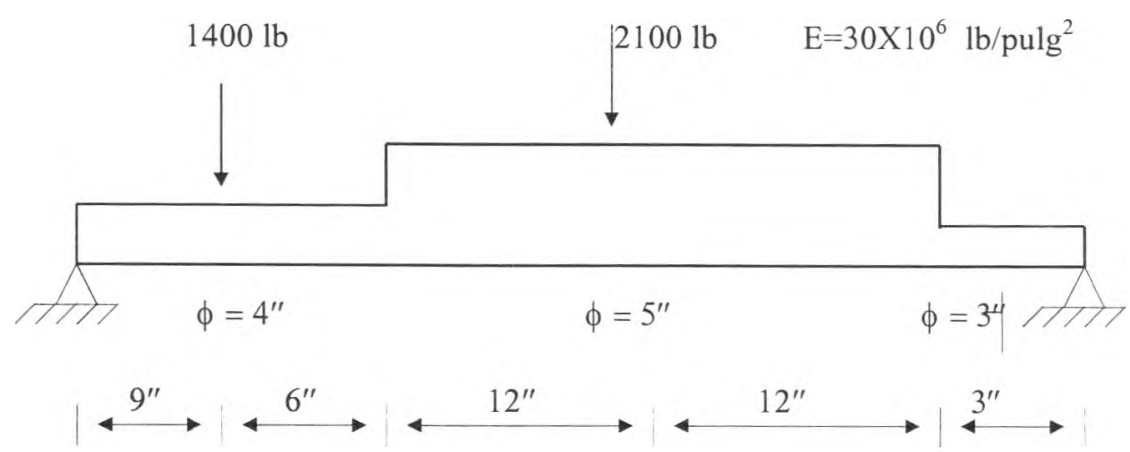

Ejemplo 1. Obténgase la elástica de esta viga.

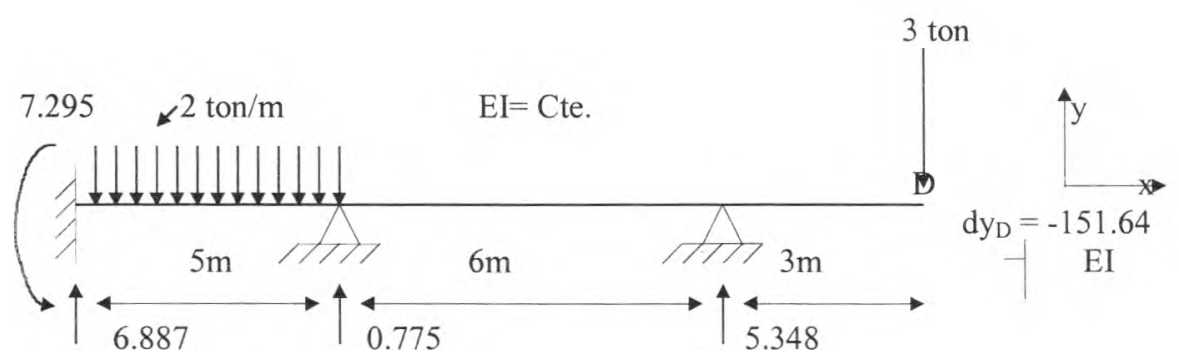

Ejemplo 2. Obténgase la elástica de esta viga, la cual se ha analizado con anterioridad mostrándose algunos resultados de interés.

Datos del ejemplo 1

\begin{tabular}{rrl}
$15,0,0$, & & \\
, 30, & 12.5664, & $30 \mathrm{e} 6$ \\
5550 & 12.5664, & $30 \mathrm{e} 6$ \\
11100 & 12.5664, & $30 \mathrm{e} 6$ \\
16650 & 12.5664, & $30 \mathrm{e} 6$ \\
18000, & 12.5664, & $30 \mathrm{e} 6$ \\
19350, & 21.6230, & $30 \mathrm{e} 6$ \\
20700, & 30.6796, & $30 \mathrm{e} 6$ \\
22050, & 30.6796, & $30 \mathrm{e} 6$ \\
23400, & 30.6796, & $30 \mathrm{e} 6$ \\
24750, & 30.6796, & $30 \mathrm{e} 6$ \\
19800, & 30.6796, & $30 \mathrm{e} 6$ \\
14850, & 30.6796, & $30 \mathrm{e} 6$ \\
9900, & 30.6796, & $30 \mathrm{e} 6$ \\
4950, & 17.3278, & $30 \mathrm{e} 6$ \\
0, & 3.9761, & $30 \mathrm{e} 6$ \\
\hline
\end{tabular}

Datos del ejemplo 2

\begin{tabular}{cc}
\hline $16,0,-151.64,1$ \\
$-7.295,1,1$ \\
$-1.408,1,1$ \\
$2.479,1,1$ \\
$4.366,1,1$ \\
$4.253,1,1$ \\
$2.140,1,1$ \\
$-0.198,1,1$ \\
$-2.536,1,1$ \\
$-4.874,1,1$ \\
$-7.212,1,1$ \\
$-9.550,1,1$ \\
$-11.888,1,1$ \\
$-8.878,1,1$ \\
$-5.868,1,1$ \\
$-2.858,1,1$ \\
$0.152,1,1$ \\
\hline \hline
\end{tabular}




\section{Solución}

Ejemplo 1. Desplazamientos perpendiculares al eje de la barra, $y(x)$

\begin{tabular}{ccrcccc}
\hline Punto & $\mathrm{x}$ & $\mathrm{M}(\mathrm{x})$ & $1(\mathrm{x})$ & $\mathrm{E}(\mathrm{x})$ & \multicolumn{1}{c}{$(\mathrm{M} / \mathrm{E} \mathrm{I}) \Delta \mathrm{x}^{2}$} & $\mathrm{y}(\mathrm{x})$ \\
\hline 1 & 0.00 & 0.00 & 12.5664 & $30,000,000.00$ & $0.000 \mathrm{E}+00$ & 0.000000 \\
2 & 3.00 & $5,550.00$ & 12.5664 & $30,000,000.00$ & $1.320 \mathrm{E}-04$ & -0.001656 \\
3 & 6.00 & $11,100.00$ & 12.5664 & $30,000,000.00$ & $2.650 \mathrm{E}-04$ & -0.003180 \\
4 & 9.00 & $16,650.00$ & 12.5664 & $30,000,000.00$ & $3.970 \mathrm{E}-04$ & -0.004439 \\
5 & 12.00 & $18,000.00$ & 12.5664 & $30,000,000.00$ & $4.300 \mathrm{E}-04$ & -0.005300 \\
6 & 15.00 & $19,350.00$ & 21.6230 & $30,000,000.00$ & $2.680 \mathrm{E}-04$ & -0.005732 \\
7 & 18.00 & $20,700.00$ & 30.6796 & $30,000,000.00$ & $2.020 \mathrm{E}-04$ & -0.005895 \\
8 & 21.00 & $22,050.00$ & 30.6796 & $30,000,000.00$ & $2.160 \mathrm{E}-04$ & -0.005856 \\
9 & 24.00 & $23,400.00$ & 30.6796 & $30,000,000.00$ & $2.290 \mathrm{E}-04$ & -0.005601 \\
10 & 27.00 & $24,750.00$ & 30.6796 & $30,000,000.00$ & $2.420 \mathrm{E}-04$ & -0.005118 \\
11 & 30.00 & $19,800.00$ & 30.6796 & $30,000,000.00$ & $1.940 \mathrm{E}-04$ & -0.004392 \\
12 & 33.00 & $14,850.00$ & 30.6796 & $30,000,000.00$ & $1.450 \mathrm{E}-04$ & -0.003473 \\
13 & 36.00 & $9,900.00$ & 30.6796 & $30,000,000.00$ & $9.680 \mathrm{E}-05$ & -0.002408 \\
14 & 39.00 & $4,950.00$ & 17.3278 & $30,000,000.00$ & $8.570 \mathrm{E}-05$ & -0.001247 \\
15 & 42.00 & 0.00 & 3.9761 & $30,000,000.00$ & $0.000 \mathrm{E}+00$ & -0.000000 \\
\hline
\end{tabular}
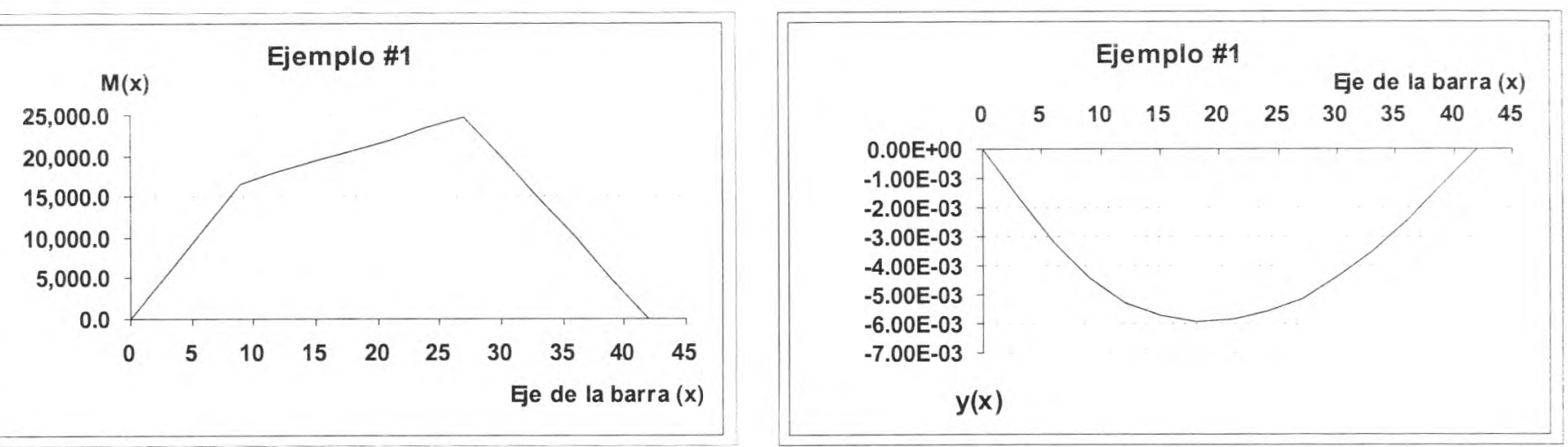

Ejemplo 2. Desplazamientos perpendiculares al eje de la barra, y(x)

\begin{tabular}{ccccccc}
\hline Punto & $\mathrm{x}$ & $\mathrm{M}(\mathrm{x})$ & $\mathrm{I}(\mathrm{x})$ & $\mathrm{E}(\mathrm{x})$ & $(\mathrm{M} / \mathrm{E} \mathrm{I}) \Delta \mathrm{x}^{2}$ & $\mathrm{y}(\mathrm{x})$ \\
\hline \hline 1 & 0.00 & -7.30 & 1.0000 & 1.00 & $-7.300 \mathrm{E}+00$ & 0.000000 \\
2 & 1.00 & -1.41 & 1.0000 & 1.00 & $-1.410 \mathrm{E}+00$ & -3.249332 \\
3 & 2.00 & 2.48 & 1.0000 & 1.00 & $2.480 \mathrm{E}+00$ & -7.906665 \\
4 & 3.00 & 4.37 & 1.0000 & 1.00 & $4.370 \mathrm{E}+00$ & -10.084997 \\
5 & 4.00 & 4.25 & 1.0000 & 1.00 & $4.250 \mathrm{E}+00$ & -7.897329 \\
6 & 5.00 & 2.14 & 1.0000 & 1.00 & $2.140 \mathrm{E}+00$ & -1.456662 \\
7 & 6.00 & -0.20 & 1.0000 & 1.00 & $-1.980 \mathrm{E}-01$ & 7.124005 \\
8 & 7.00 & -2.54 & 1.0000 & 1.00 & $-2.540 \mathrm{E}+00$ & 15.506673 \\
9 & 8.00 & -4.87 & 1.0000 & 1.00 & $-4.870 \mathrm{E}+00$ & 21.353340 \\
10 & 9.00 & -7.21 & 1.0000 & 1.00 & $-7.210 \mathrm{E}+00$ & 22.326008 \\
11 & 10.00 & -9.55 & 1.0000 & 1.00 & $-9.550 \mathrm{E}+00$ & 16.086676 \\
\hline & & & & & & continúa...
\end{tabular}


Una aplicacion de la solución de sistemas de ecuaciones lineales no homogéneas...

Ejemplo 2. Desplazamientos perpendiculares al eje de la barra, y (x) (continuación)

\begin{tabular}{|c|c|c|c|c|c|c|}
\hline Punto & $\mathrm{x}$ & $\mathrm{M}(\mathrm{x})$ & $\mathrm{I}(\mathrm{x})$ & $\mathrm{E}(\mathrm{x})$ & $(M / E I) \Delta x^{2}$ & $y(x)$ \\
\hline 12 & 11.00 & -11.89 & 1.0000 & 1.00 & $-1.190 \mathrm{E}+01$ & 0.297344 \\
\hline 13 & 12.00 & -8.88 & 1.0000 & 1.00 & $-8.880 \mathrm{E}+00$ & -.27 .379988 \\
\hline 14 & 13.00 & -5.87 & 1.0000 & 1.00 & $-5.870 \mathrm{E}+00$ & -63.935322 \\
\hline 15 & 14.00 & -2.86 & 1.0000 & 1.00 & $-2.860 \mathrm{E}+00$ & -106.358658 \\
\hline 16 & 15.00 & 0.15 & 1.0000 & 1.00 & $1.520 \mathrm{E}+01$ & -151.639999 \\
\hline
\end{tabular}
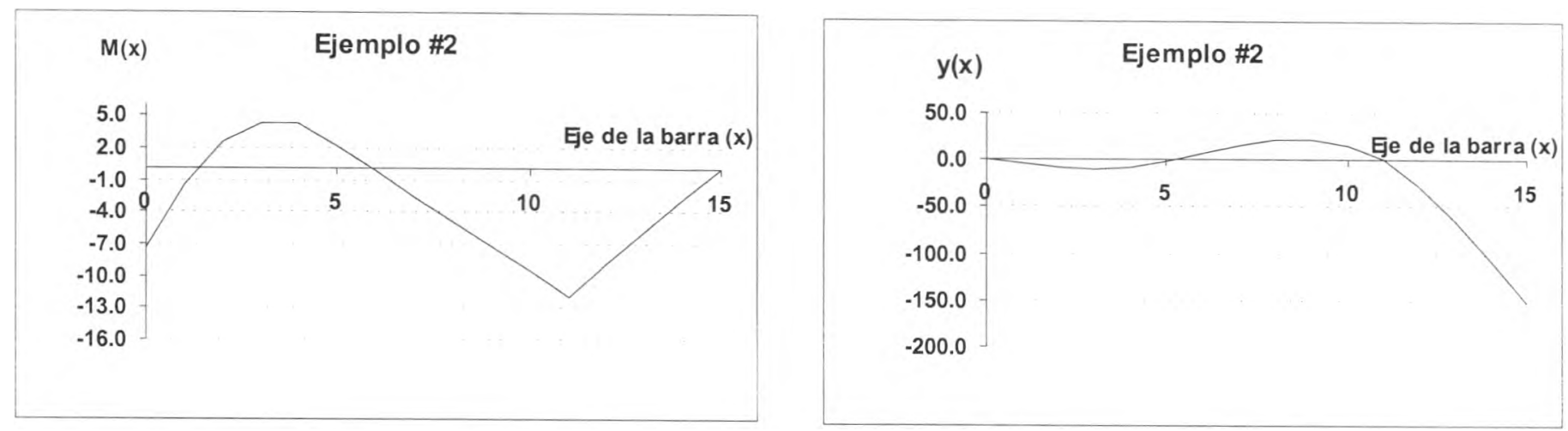

\section{Ejemplo de comparación}

Desplazamientos perpendiculares al eje de la barra, $y(x)$

$$
\begin{array}{lr}
\text { w }= & -2 \\
\mathrm{~L}= & 42 \\
\mathrm{I}= & 1 \\
\mathrm{E}= & 1
\end{array}
$$

\begin{tabular}{|c|c|c|c|c|c|c|c|c|c|}
\hline Punto & $\mathrm{x}$ & $M(x)$ & Excata & $\Delta \mathrm{x}=6$ & $(1-2) / 1 \times 100$ & $\begin{array}{c}y(x) \\
\Delta x=3\end{array}$ & $(1-3) / 1 \times 100$ & $\Delta \mathrm{x}=1$ & $(1-4) / 1 \times 100$ \\
\hline & & & $(1)$ & (2) & & (3) & & (4) & \\
\hline 1 & 0.0 & -441.0 & 0.0 & 0.0 & 0.0 & 0.0 & 0.0 & 0.0 & 0.0 \\
\hline 2 & 3.0 & -292.5 & $-1,755.0$ & & & $-1,842.7$ & -5.0 & $-1,764.7$ & -0.6 \\
\hline 3 & 6.0 & -162.0 & $-6,156.0$ & $-6,804.0$ & -10.5 & $-6,318.0$ & -2.6 & $-6,174.0$ & -0.3 \\
\hline 4 & 9.0 & -49.5 & $-12,028.5$ & & & $-12,251.2$ & -1.9 & $-12,053.2$ & -0.2 \\
\hline 5 & 12.0 & 45.0 & $-18,360.0$ & $-19,440.0$ & -5.9 & $-18,630.0$ & -1.5 & $-18,390.0$ & -0.2 \\
\hline 6 & 15.0 & 121.5 & $-24,300.0$ & & & $-24,603.7$ & -1.2 & $-24,333.7$ & -0.1 \\
\hline 7 & 18.0 & 180.0 & $-29,160.0$ & $-30,456.0$ & -4.4 & $-29,484.0$ & -1.1 & $-29,196.0$ & -0.1 \\
\hline 8 & 21.0 & 220.5 & $-32,413.5$ & & & $-32,744.2$ & -1.0 & $-32,450.2$ & -0.1 \\
\hline 9 & 24.0 & 243.0 & $-33,696.0$ & $-34,992.0$ & -3.8 & $-34,020.0$ & -1.0 & $-33,732.0$ & -0.1 \\
\hline 10 & 27.0 & 247.5 & $-32,805.0$ & & & $-33,108.8$ & -0.9 & $-32,838.7$ & -0.1 \\
\hline 11 & 30.0 & 234.0 & $-29,700.0$ & $-30,780.0$ & -3.6 & $-29,970.0$ & -0.9 & $-29,730.0$ & -0.1 \\
\hline 12 & 33.0 & 202.5 & $-24,502.5$ & & & $-24,725.3$ & -0.9 & $-24,527.2$ & -0.1 \\
\hline 13 & 36.0 & 153.0 & $-17,496.0$ & $-18,144.0$ & -3.7 & $-17,658.0$ & -0.9 & $-17,514.0$ & -0.1 \\
\hline 14 & 39.0 & 85.5 & $-9,126.0$ & & & $-9,213.8$ & -1.0 & $-9,135.7$ & -0.1 \\
\hline 15 & 42.0 & 0.0 & 0.0 & 0.0 & 0.0 & 0.0 & 0.0 & 0.0 & 0.0 \\
\hline
\end{tabular}




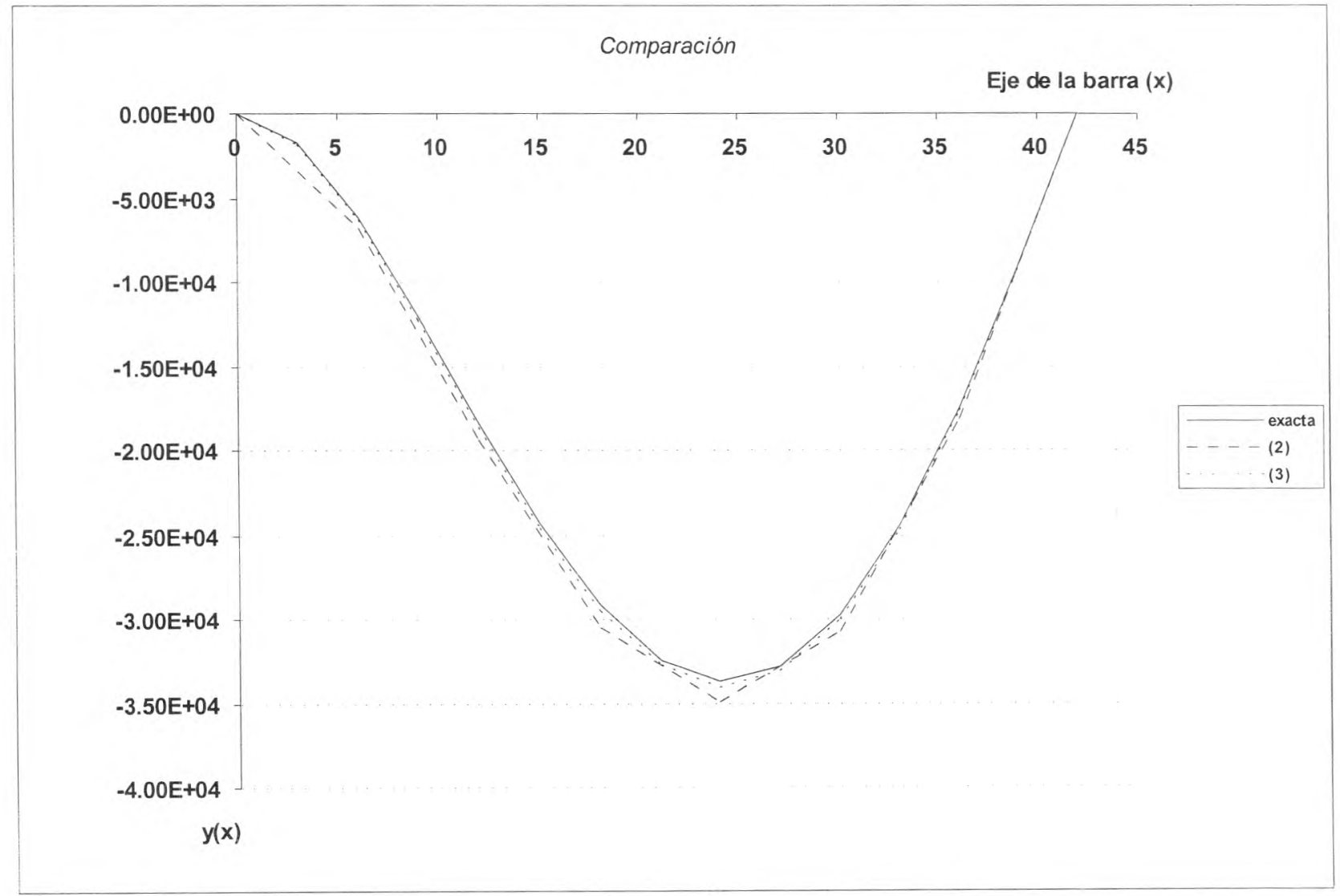

DECLARE SUB gauss ( $\mathrm{n} \%, \mathrm{ab} \%, \mathrm{a} !(), \mathrm{b} !(), \mathrm{x} !())$

DECLARE SUB titulo (ir\%, encab\$, ncol\%)

DECLARE SUB dibuja ( $m$ ! (), npun $\%$, ivx $1 \%$, ivy $1 \%$, ivx $2 \%$, ivy $2 \%$, deltx!, encab\$, ir $\%$, f 1 !)

programa: ELAS3.BAS

' objetivo : Obtención y graficación de la elástica de barras de eje recto

‘ Autor : Ing. Fernando Monroy Miranda

- Fecha : Junio de 1996

DEFINT I-K, N

DEF fnsigno $(x, i z)$

ial $=\operatorname{SGN}(\mathrm{x})$

IF iz $>0$ THEN

IF ial $=0$ THEN ial $=1$

ELSE

IF ia $1=0$ THEN ia $1=-1$

END IF

fnsigno $=\mathrm{ia} 1$

END DEF

DIM a $(50,50), y(50), m(50), e(50), x i(50), \operatorname{mei}(52), x(50)$

' Lectura de datos de archivo

INPUT "Nombre completo del archivo donde están los datos "; arch\$

OPEN $\operatorname{arch} \$$ FOR INPUT AS \# 1

INPUT \# 1 , nom\$ 
INPUT \# 1, npun, y1, ynpun, deltx

$\mathrm{d} 2=\operatorname{deltx} * \operatorname{delt} \mathrm{x}$

FOR $i=1$ TO npun

INPUT \# $1, \mathrm{~m}(\mathrm{i}), \mathrm{xi}(\mathrm{i}), \mathrm{e}(\mathrm{i})$

$\operatorname{mei}(\mathrm{i})=\mathrm{m}(\mathrm{i}) * \mathrm{~d} 2 /(\mathrm{e}(\mathrm{i}) * \mathrm{xi}(\mathrm{i}))$

NEXT i

CLOSE \# 1

'- - formación de la matriz de coeficientes y del vector de términos independientes

necs $=$ npun -2

FOR $\mathrm{i}=1 \mathrm{TO}$ necs

$\mathrm{a}(\mathrm{i}, \mathrm{i})=1$ !

IF $\mathrm{i}>1$ THEN a $(\mathrm{i}, \mathrm{i}-1)=-.5$

IF $\mathrm{i}<\operatorname{necs}$ THEN a $(\mathrm{i}, \mathrm{i}+1)=-.5$

$y(i)=-\operatorname{mei}(i+1) / 2$

NEXT i

$y(1)=y(1)+y 1 / 2$

$y($ necs $)=y($ necs $)+y$ npun $/ 2$

‘- - solución del sistema de ecuaciones utilizando Gauss

CALL gauss(necs, 2, a (), y(), x())

'- mostrando desplazamientos

\section{CLS}

CALL titulo (1, nom\$, 80)

LOCATE 3, 15: PRINT "Desplazamientos perpendiculares al eje de la barra y $(x)$ "

PRINT

PRINT "Punto $\mathrm{x} \quad \mathrm{M}(\mathrm{x}) \quad \mathrm{I}(\mathrm{x}) \quad \mathrm{E}(\mathrm{x}) \quad(\mathrm{M} / \mathrm{EI})$ ëxý $\quad \mathrm{y}(\mathrm{x})$ "

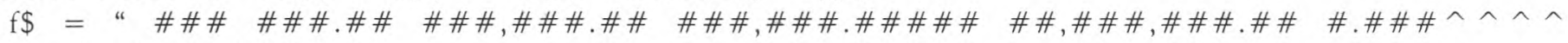
\#,\#\#\#.\#\#\#\#\#\#"

PRINT USING $\mathrm{f} \$ 1 ; ; 0 ; \mathrm{m}(1) ; \mathrm{xi}(1) ; \mathrm{e}(1) ; \operatorname{mei}(1) ; \mathrm{y} 1$

FOR $i=1$ TO necs

$\mathrm{k}=\mathrm{i}+\mathrm{l}$

PRINT USING $\mathrm{f} \$ \mathrm{k} ; \mathrm{i} * \operatorname{deltx} ; \mathrm{m}(\mathrm{k}) ; \mathrm{xi}(\mathrm{k}) ; \mathrm{e}(\mathrm{k}) ; \operatorname{mei}(\mathrm{k}) ; \mathrm{x}(\mathrm{i})$

NEXT i

PRINT USING f\$; npun; (npun - 1) *deltx; m(npun); xi(npun); e(npun); mei(npun); ynpun

PRINT "Para continuar presione cualquier tecla": $\mathrm{t} \$=\operatorname{INPUT} \$(1)$

gráficas

CALL dibuja(m(), npun, 10, 89, 309, 9, deltx, "Diagrama de momentos flexionantes", 13, .05)

CALL titulo(1, nom\$, 40)

$\operatorname{mei}(1)=y 1$

mei(npun) $=$ ynpun

FOR $\mathrm{i}=1 \mathrm{TO}$ necs

$\operatorname{mei}(\mathrm{i}+1)=\mathrm{x}(\mathrm{i})$

NEXT i

CALL dibuja(mei(), npun, 10, 189, 309, 109, deltx, "E L A S T I C A", 25, .05)

$\mathrm{t} \$=\operatorname{INPUT} \$(1)$ 
END

SUB dibuja (m(), npun, ivx1, ivy1, ivx2, ivy2, deltx, encab\$, ir, f1) STATIC

'búsqueda de máximos, abriendo ventana

$\operatorname{mmax}=-1 \mathrm{E}+37$

$\operatorname{mmin}=1 \mathrm{E}+37$

FOR $\mathrm{j}=1 \mathrm{TO}$ npun

IF $m(j)>\operatorname{mmax}$ THEN $\operatorname{mmax}=m(j)$

IF $m(j)<\operatorname{mmin}$ THEN $m$ min $=m(j)$

NEXT $j$

\section{SCREEN 1}

VIEW (ivx1, ivy1)-(ivx2, ivy2), , 2

lon $=($ npun -1$) *$ delt $x$

IF ABS $(\operatorname{mmin})>\operatorname{ABS}(\operatorname{mmax})$ THEN $\operatorname{maxy}=\mathrm{ABS}(\operatorname{mmin})$ ELSE $\operatorname{maxy}=\mathrm{ABS}(\operatorname{mmax})$

yinc $=\mathrm{f} 1 * \operatorname{maxy}$

WINDOW $(-\mathrm{f} 1 *$ lon, $\operatorname{mmin}+$ fnsigno $(\operatorname{mmin},-1) *$ yinc $)-(\operatorname{lon} *(1 !+\mathrm{f} 1), \operatorname{mmax}+$ fnsigno $(\operatorname{mmax}, 1) *$ yinc $)$

‘ejes horizontal y vertical

$\operatorname{LINE}(0,0)-($ lon, 0$)$

$\operatorname{LINE}(0, \mathrm{mmin})-(0, \operatorname{mmax})$

' dibujo del diagrama

$\operatorname{PSET}(0, \mathrm{~m}(1))$

FOR $\mathrm{i}=2 \mathrm{TO}$ npun

LINE - (deltx * (i - 1), m(i)), 1

NEXT i

CALL titulo(ir, encab\$, 40)

END SUB

\section{DEFINT L-M}

SUB gauss $(n, a b \%, a(), b(), x())$ STATIC

$\mathrm{nab}=\mathrm{n}-\mathrm{ab} \%$

FOR $\mathrm{i}=1 \mathrm{TO}$ nab

$\mathrm{i} 1=\mathrm{i}+1$

$i a b=i+a b \%$

aii $=\mathrm{a}(\mathrm{i}, \mathrm{i})$

FOR k = i1 TO iab

FOR j = il TO iab

$$
a(k, j)=a(k, j)-a(k, i) * a(i, j) / a i i
$$

NEXT $\mathrm{j}$

$\mathrm{b}(\mathrm{k})=\mathrm{b}(\mathrm{k})-\mathrm{a}(\mathrm{k}, \mathrm{i}) * \mathrm{~b}(\mathrm{i}) / \mathrm{aii}$

\section{NEXT k}

NEXT i

$n a b=n a b+1$

$\mathrm{n} 1=\mathrm{n}-1$ 


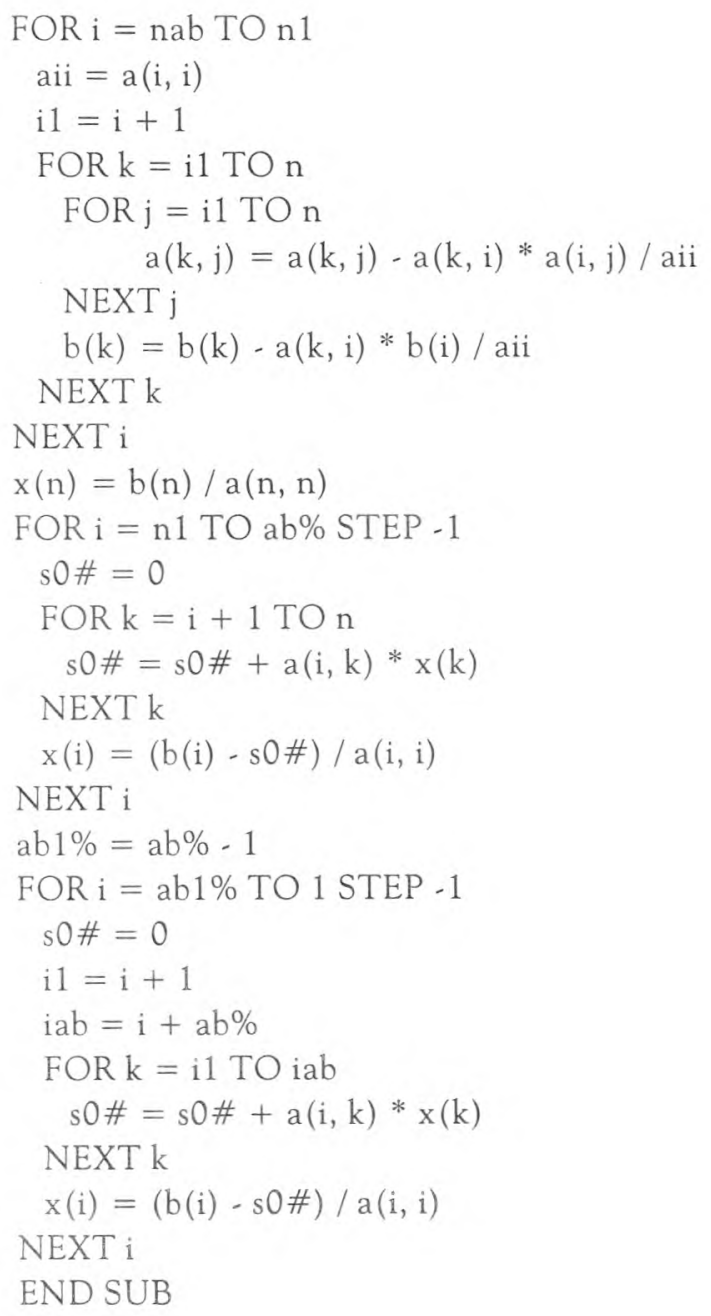

DEFSNG L-M

SUB titulo (ir, encab\$, ncol) STATIC

$\mathrm{k}=\mathrm{LEN}($ encab\$)

IF $\mathrm{k}>\mathrm{ncol}$ THEN $\mathrm{k}=\mathrm{ncol}$

ic $=($ ncol $-\operatorname{LEN}(\operatorname{LEFT} \$($ encab\$, k $))) / 2$

IF ic $<\underline{\underline{0 \text { THEN ic }=1}}$

LOCATE ir, ic: PRINT LEFT\$(encab\$, k);

END SUB

\section{Comparación con la solución exacta}

Para una viga empotrada en su extremo izquierdo y simple. mente apoyada en el extremo derecho, sujeta a una carga uniformemente distribuida en toda su longitud $\mathbf{L}$, con propiedades geométricas y elásticas constantes, la solución exacta (considerando deformaciones pequeñas y sólo producidas por la flexión) para el momento flexionante $\mathrm{M}(\mathrm{x})$ y la curva elástica $\mathrm{y}(\mathrm{x})$ es

$$
\begin{gathered}
M(x)=w\left(4 x^{2}-5 L x+L^{2}\right) / 8 \\
y(x)=w\left(2 x^{4}-5 L x^{3}+3 L^{2} x^{2}\right) / 48 E I
\end{gathered}
$$

Para este caso, se prepararon los datos correspondientes a $\Delta \mathrm{x}=\mathrm{L} / 7, \mathrm{~L} / 14$ y L/42 $(\mathrm{L}=$ longitud de la viga) y con ellos se alimentó el programa incluido en este trabajo, en donde se muestran, tanto los resultados obtenidos con la aplicación del programa como la solución exacta de la viga cuyas características se describieron en el párrafo anterior. Como puede verse, aún para un $\Delta \mathrm{x}$ grande $(\mathrm{L} / 7)$ el error promedio 
es de $4 \%$, que para ciertos casos prácticos pudiera considerarse aceptable, para $\Delta x=L / 14$ y L/42 el error disminuye aproximadamente a 1.3 y $.2 \%$ respectivamente.

\section{Conclusiones}

El método utilizado para resolver la ecuación (1) es un método de tercer orden, lo que significa que produce resultados "exactos" (seleccionando adecuadamente el número de puntos sobre la barra, $\Delta x$ pequeño alrededor de $\mathrm{L} / 10 \mathrm{a} / \mathrm{L} / 15$ ) si la función $\mathrm{y}(\mathrm{x})$ es un polinomio de tercer grado o menor, para nuestro problema significaría que sobre la barra estuvieran actuando sólo fuerzas o momentos concentrados; sin embargo, en un buen número de casos existen fuerzas actuando con cierta ley de variación (uniformes, lineales, etc.), lo anterior trae como consecuencia que el grado de $\mathrm{y}(\mathrm{x})$ sea mayor que 3 , en tales condiciones se pueden esperar resultados más precisos si se utiliza un método numérico de mayor orden (quinto o séptimo). No obstante, en la mayoría de problemas de ingeniería, las hipótesis de análisis, así como el grado de precisión requerido en las soluciones, no justifica el uso de procedimientos de mayor óden, de manera que, el método de tercer orden resulta adecuado para la solución de este problema.

Aunque el programa fue desarrollado en una versión de BASIC, con poco esfuerzo, todo o una parte de éste puede ser traducido a otro lenguaje (si es necesario), o bien, modificarlo para incorporarlo a algún programa de análisis estructural con lo que se tendrá la posibilidad de dibujar la elástica de la estructura como una opción más dentro del mismo programa.

En cuanto a la solución del sistema de ecuaciones, el método de Gauss se puede modificar para considerar la simetría de la matriz de coeficientes como lo hace el método de Cholesky, el cual también se pudo utilizar.

\section{Referencias}

Beaufait F.W. (1977). Basic Concepts of Structural Analysis. Prentice Hall.

Gere J.M y Timoshenko S.P. (1986). Resistencia de materiales. GEI, 2a. Ed.

Laible J.P. (1985). Structural Analysis. CBS College Publishing

Marshall N. (1990). Structures. Third edition, Longman Scientific and Technical.

Popov E. (1972). Introducción a la mecánica de sólidos. LIMUSA. México.

"QUICK-BASIC", versiones 2.0, 4.0 y 4.5 Microsoft Corp. 1984 a 1989.

Sánchez J.L. y Franco V. (1996). Solución de sistemas de ecuaciones lineales no homogéneas con matriz en banda. Revista Ingeniería, Vol. LXVI, No. 2, abriljunio.

Smith, Wolford J.C. y James M.L. (1976). Métodos numéricos aplicados a la computación digital con Fortran Gerald M. RSI, México.

\section{Semblanza del autor}

Fernando Monroy-Miranda. Egresó como ingeniero mecánico de la Facultad de Ingeniería, UNAM. Imparte las asisignaturas de mecánica de materiales I, II y III, teoría general de las estructuras, así como estructuras isostáticas y análisis estructural, de las cuales actualmente es coordinador. Ocupó el cargo de jefe de laboratorio de materiales y ha impartido cursos sobre análisis estructural y uso de programas de computadora para análisis y diseño de estructuras en el Colegio de Ingenieros Civiles de México, las Sociedades Mexicanas de Ingeniería Sísmica y Estructural, así como en la División de Educación Continua de la Facultad de Ingeniería de la UNAM, la Comisión Federal de Electricidad y en algunas empresas del sector privado. 
DOI: http://dx.doi.org/10.22201/fi.25940732e.2001.02n2.007 